\title{
Thermal-solutal flows and segregation and their control by angular vibration in vertical Bridgman crystal growth
}

\author{
Y.C. Liu ${ }^{\text {a }}$,W.C. Yu ${ }^{\text {b }}$, B. Roux ${ }^{\text {c }}$, T.P. Lyubimova ${ }^{\text {d }}$, C.W. Lan ${ }^{\mathrm{a}, *}$ \\ ${ }^{a}$ Department of Chemical Engineering, National Taiwan University, Taipei 10617, Taiwan, ROC \\ ${ }^{\mathrm{b}}$ Department of Molecular Science and Engineering, National Taipei University of Technology, Taiwan, ROC \\ ${ }^{\mathrm{c}}$ Laboratoire Modélisation et simulation numérique en mécanique, L3M: CNRS-Universités d'Aix-Marseille, France \\ ${ }^{\mathrm{d}}$ Institute of Continuous Media Mechanics UB RAS, Perm, Russia
}

Received 18 July 2006; received in revised form 1 September 2006; accepted 1 September 2006

Available online 8 September 2006

\begin{abstract}
Thermal-solutal flows and their induced segregation and supercooling are important in the growth of an alloy crystal. For the vertical Bridgman crystal growth having a stabilized thermal profile, in addition to the thermal convection induced by radial thermal gradients, solute gradients can either induce or suppress the flow depending on the solute density. Such thermal-solutal flows significantly affect the segregation behavior, constitutional supercooling, and thus the morphological instability of the solidification front. A transparent Bridgman growth of succinonitrile containing a lighter (acetone) or heavier (salol) solute was investigated. The evolution of the interface shape as well as morphological instability was visualized and was interpreted through computer simulation. To further control the flow and segregation, angular vibration was applied and its effects on the thermal-solutal flows and morphological instability were investigated.
\end{abstract}

(C) 2006 Elsevier Ltd. All rights reserved.

PACS: 44.25.+f; 47.27.Te; 81.10.Fq; 02.60.c6; 02.70.Fj

Keywords: Thermal-solutal flows; Angular vibration; Convection; Morphology; Segregation; Bridgman crystal growth

\section{Introduction}

The vertical Bridgman or gradient freeze technique is a popular method for crystal growth, refining, and purification. Particularly, its simplicity in operation and the low thermal gradients are very suitable to the growth of compound semiconductor crystals (Brice, 1986; Monberg, 1994). The stabilized thermal configuration is also a great advantage offering weak convection and less growth striations due to the stable flow. Nevertheless, for the growth of an alloy crystal, the solutal gradients could induce solutal convection or suppress thermal convection depending on the density of the solute (Adornato and Brown, 1987; Lan and Chen, 1996). In some cases, the interaction of the thermal-solutal flows could lead to flow instability (Ghorayeb et al., 1999; Zhou and Zebib, 1994; Nishimura

\footnotetext{
* Corresponding author. Tel./fax: +886223633917.

E-mail address: cwlan@ntu.edu.tw (C.W. Lan).
}

et al., 1998; Shi and Lu, 2006). Because of the lack of active melt stirring, the thermal-solutal flows sometimes cause large radial and axial uniformities due to the poor mixing and solute segregation. More importantly, the morphological instability could be easily induced by constitutional supercooling (Tiller et al., 1953; Mullins and Sekerka, 1964) as a result of local solute accumulation (Singh et al., 1996; Lan and Tu, 2001). Therefore, a better understanding of the flow behavior and finding an active control mean over the melt convection are useful for this process (Lan, 2004). For example, the accelerated crucible rotation technique (ACRT) (Scheel, 1971) and angular vibration method (Yu et al., 2004) are particularly effective. In a recent study (Lan, 2005), the angular vibration seems to be more effective in radial segregation control, as compared with ACRT, while no significant flow oscillations are induced.

In this paper, we present the visualized morphological evolution during a transparent Bridgman growth of succinonitrile (SCN) containing acetone (a lighter solute) or salol (a heavier 


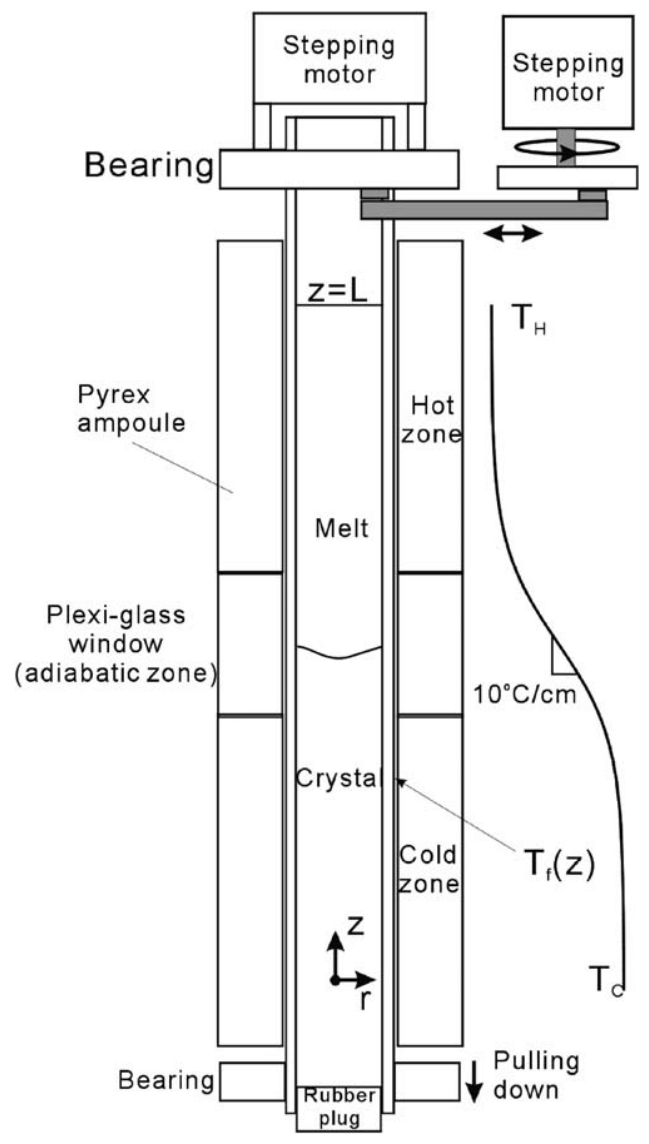

Fig. 1. Sketch of the experimental setup for vertical Bridgman crystal growth with angular vibration; the furnace profile $T_{f}(z)$ was used for simulation.

solute) to illustrate the effects of the thermal-solutal flow on the interface shape and morphological instability. The angular vibration technique was also adopted to control the flows, and thus the segregation and the interface morphology. To explain the observed morphological transition, computer simulation was further conducted.

In the next section, the experimental setup and solidification experiments are described. Section 3 is devoted to the numerical model. The results and discussion are arranged in Section 4 before a short conclusion is given.

\section{Experimental}

SCN containing about $0.064 \mathrm{wt} \%$ acetone or $0.15 \mathrm{wt} \%$ salol was directionally solidified in a transparent vertical Bridgman system, as sketched in Fig. 1. The use of the higher salol concentration was due to its higher segregation coefficient $K(0.16$ for salol and 0.1 for acetone). Before experiments, SCN (Acros, Inc., about $99 \%$ purity) was purified first by vacuum distillation at $50 \mathrm{~m}$ torr for five times. The distilled sample, collected in a $17-\mathrm{mm}$ diameter Pyrex ampoule $(2.5 \mathrm{~mm}$ in thickness), was further purified by three-zone refining for more than 60 passes. The purified sample was then examined by directional solidification. No morphological breakdown was observed up to $7 \mu \mathrm{m} / \mathrm{s}$ of the solidification speed for a thermal gradient of
$8-10 \mathrm{~K} / \mathrm{cm}$. To perform crystal growth experiments, acetone or salol was injected into the sample through a $5 \mu \mathrm{l}$ micro-syringe inserted into the bottom of the sample. The total sample length was about $20 \mathrm{~cm}$.

The furnace for directional solidification consisted of two heating zones made of copper blocks each with a Nichrome wire inside as a heating element. In between, a transparent insulation zone made of Plexiglas was used. The hot- and coldzone temperatures were controlled independently by two PID controllers and the temperatures were set to $80^{\circ} \mathrm{C}$ (top) and $40^{\circ} \mathrm{C}$ (bottom), respectively. The thermal gradient at the interface was measured by an immersed thermocouple traveling with the sample. By taking an average of the gradients at the interface from solidification and melting curves, the thermal gradient was measured being about $8-10 \mathrm{~K} / \mathrm{cm}$. To translate the ampoule accurately, a microstepping motor was used to drive a screw slide; the translation rate was controlled at $1.6 \mu \mathrm{m} / \mathrm{s}$ in this study. During solidification, a digital video camera was used to record the evolution of the interface morphology with a back lighting to enhance the contrast of the image. Also, to allow a smooth rotational motion, the ampoule was tightly fitted into a pair of bearings (top and bottom) that were both mounted on the translating system. Furthermore, to generate angular vibration, as shown in Fig. 1, a stepping motor was used to rotate a disk having a shaft connected to the other disk that mounted the sample. The vibration amplitude was controlled by the position of the shaft in the disk, while the frequency was controlled by the rotation speed of the stepping motor. In the experiments, the dimensionless amplitude was about 0.045 , i.e., a fraction of $2 \pi$.

\section{Governing equations and numerical solution}

The vertical Bridgman crystal growth depicted in Fig. 1 is simulated, where the furnace environment is described by an effective heating profile $T_{f}(z, t)$. To start crystal growth from a stationary state (without ampoule movement) in simulation, this profile is moved upward at speed $U_{h}$. In the experiments, the furnace was kept stationary, while the ampoule was moving downward at a given speed after the growth started. The angular vibration is applied in the azimuthal angle direction with an amplitude $b_{v}$ (a fraction of $2 \pi$ ) and angular frequency $\Omega$ (or frequency $f$ in Hertz); $\Omega=2 \pi f$. Because the heating was rather uniform and the observed interface shape was nearly symmetric, the system for simulation is assumed axisymmetric. Accordingly, the flow and temperature fields, as well as the growth front (the melt/crystal interface, $h_{c}(r, t)$ ). The melt is further assumed incompressible and Newtonian, while the flow is laminar. The Boussinesq approximation is also adopted. Dimensionless variables are defined by scaling length with the crystal diameter $R_{c}$, time $t$ with $R_{c}^{2} / \alpha_{m}$, velocity with $\alpha_{m} / R_{c}$, temperature with the melting point $T_{m}$, and solute concentration by the initial concentration $C_{0}$, where $\alpha_{m}$ is the thermal diffusivity of the melt. For the convenience of representation, all the variables defined afterwards with a superscript $*$ are dimensionless unless otherwise stated. The governing equations for the time-dependent fluid flow and heat and mass transfer in 
terms of dimensionless stream function $\Psi^{*}$, vorticity $\omega^{*}$, azimuthal velocity $v_{\theta}^{*}$, temperature $T^{*}$ and solute concentration $C^{*}$ can be written as the following:

Equation of motion:

$$
\begin{aligned}
\frac{\partial \omega^{*}}{\partial t^{*}} & +\frac{\partial}{\partial r^{*}}\left(\frac{\omega^{*}}{r^{*}} \frac{\partial \psi^{*}}{\partial z^{*}}\right)-\frac{\partial}{\partial z^{*}}\left(\frac{\omega^{*}}{r^{*}} \frac{\partial \psi^{*}}{\partial r^{*}}\right) \\
+ & \operatorname{Pr}\left[\frac{\partial}{\partial r^{*}}\left(\frac{1}{r^{*}} \frac{\partial}{\partial r^{*}}\left(r^{*} \omega^{*}\right)\right)+\frac{\partial}{\partial z^{*}}\left(\frac{1}{r^{*}} \frac{\partial}{\partial z^{*}}\left(r^{*} \omega^{*}\right)\right)\right] \\
+ & \frac{\partial}{\partial z}\left(\frac{v_{\theta}^{2}}{r}\right)-\operatorname{Pr} R a_{T} \frac{\partial T^{*}}{\partial r^{*}}+\operatorname{Pr} \operatorname{Ra}_{S} \frac{\partial C^{*}}{\partial r^{*}}=0 .
\end{aligned}
$$

Stream equation:

$\frac{\partial}{\partial z^{*}}\left(\frac{1}{r^{*}} \frac{\partial \psi^{*}}{\partial z^{*}}\right)+\frac{\partial}{\partial r^{*}}\left(\frac{1}{r^{*}} \frac{\partial \psi^{*}}{\partial r^{*}}\right)+\omega^{*}=0$.

Circulation equation:

$$
\begin{aligned}
& -\frac{\partial v_{\theta}^{*}}{\partial t^{*}}+\frac{1}{r^{* 2}}\left[\frac{\partial}{\partial r^{*}}\left(r^{*} v_{\theta}^{*} \frac{\partial \psi^{*}}{\partial z^{*}}\right)-\frac{\partial}{\partial z^{*}}\left(r^{*} v_{\theta}^{*} \frac{\partial \psi^{*}}{\partial r^{*}}\right)\right] \\
& +\operatorname{Pr}\left[\frac{\partial}{\partial r^{*}}\left(\frac{1}{r^{*}} \frac{\partial\left(r^{*} v_{\theta}^{*}\right)}{\partial r^{*}}\right)+\frac{\partial}{\partial z^{*}}\left(\frac{1}{r^{*}} \frac{\partial\left(r^{*} v_{\theta}^{*}\right)}{\partial z^{*}}\right)\right]=0 .
\end{aligned}
$$

Energy equation:

$$
\begin{aligned}
& -\frac{\partial T^{*}}{\partial t^{*}}-\frac{\partial}{\partial r^{*}}\left(r^{*} u^{*} T^{*}\right)-\frac{\partial}{\partial z^{*}}\left(r^{*} v^{*} T^{*}\right) \\
& +\frac{\partial}{\partial z^{*}}\left(r^{*} \alpha_{i}\left(T^{*}\right) \frac{\partial T^{*}}{\partial z^{*}}\right) \\
& +\frac{\partial}{\partial r^{*}}\left(r^{*} \alpha_{i}\left(T^{*}\right) \frac{\partial T^{*}}{\partial r^{*}}\right)=0, \quad i=(m, c) .
\end{aligned}
$$

Solute equation:

$$
\begin{aligned}
& -\frac{\partial C^{*}}{\partial t^{*}}-\frac{\partial}{\partial r^{*}}\left(r^{*} u^{*} C^{*}\right)-\frac{\partial}{\partial z^{*}}\left(r^{*} v^{*} C^{*}\right) \\
& +\frac{P r}{S c}\left[\frac{\partial}{\partial z^{*}}\left(r^{*} \frac{\partial C^{*}}{\partial z^{*}}\right)+\frac{\partial}{\partial r^{*}}\left(r^{*} \frac{\partial C^{*}}{\partial r^{*}}\right)\right]=0 .
\end{aligned}
$$

The solute diffusion in the solid phase is neglected. In the above equations, $\operatorname{Pr}$ is the Prandtl number $\left(\operatorname{Pr} \equiv v_{m} / \alpha_{m}\right)$, where $v_{m}$ is the kinematic melt viscosity, $S c$ the Schmidt number $(S c \equiv$ $v_{m} / D$ ), and $D$ the solute diffusivity in the melt. Also, $\alpha_{i}$ is the thermal diffusivity of phase $i ; i=c$ for the crystal and $m$ for the melt. Two important dimensionless variables, $R a_{T}$ and $R a_{S}$, in the source term of the equation of motion are defined as follows:

$R a_{T} \equiv \frac{g_{0} \beta_{T} T_{m} R_{C}^{3}}{\alpha_{m} v_{m}}, \quad R a_{S} \equiv \frac{g_{0} \beta_{S} C_{0} R_{C}^{3}}{\alpha_{m} v_{m}}$,

where $g$ is the gravitational acceleration and $\beta_{T}$ and $\beta_{S}$ are the thermal and solutal expansion coefficients, respectively. The stream function $\psi^{*}$ and vorticity $\omega^{*}$ in the above equations are defined in terms of the radial $\left(u^{*}\right)$ and axial $\left(v^{*}\right)$ velocities as

$u^{*}=-\frac{1}{r^{*}} \frac{\partial \psi^{*}}{\partial z^{*}}, \quad v^{*}=\frac{1}{r^{*}} \frac{\partial \psi^{*}}{\partial r^{*}}$, $\omega^{*}=\frac{\partial u^{*}}{\partial z^{*}}-\frac{\partial v^{*}}{\partial r^{*}}$

To solve the above governing equations, boundary conditions are also required. Most of the boundary conditions for melt flow and heat and mass transfer can be found elsewhere (Lan and Liang, 1998). In short, the energy and solute conservation are applied to the growth interface. At the ampoule/material interfaces, heat flux continuity is forced, while the solute flux is set to zero. The melt surface is set to be stress free. The no-slip boundary condition is used for the solid boundaries of the interface. Therefore, the angular vibration of the crucible can be described by the sinusoidal azimuthal velocity as the following:

$v_{\theta}^{*}=2 \pi b_{v} f^{*} r^{*} \sin \left(2 \pi f^{*} t^{*}\right)$,

where $2 \pi b_{v}$ is the angular vibration amplitude and $f^{*}$ the dimensionless vibration frequency scaled by $\alpha_{m} / R_{c}^{2}$. Because the solidification time in experiments last for hours, it is too time consuming to perform direct numerical simulation (DNS) using Eq. (8) as the boundary condition for high-frequency vibrations. Instead, if the frequency is high such that the thickness of viscous boundary layer is small in comparison with all characteristics, an averaged flow approach using the Schlichting boundary layer approximation (SBLA) is possible. In other words, if the Schlichting boundary layer is very thin, we can take this slip tangential velocity $\left(u_{t}^{*}\right)$ for the growth front as the following (Yu et al., 2006):

$u_{t}^{*}=\frac{b_{v}^{2}\left(\pi f^{*}\right) r^{*}}{2} \sin (\varphi)$,

where $\varphi$ is the angle between the tangent to the growth front and the axis of vibration axis. With this boundary condition, the simulation cost for angular vibration is greatly reduced because the time step for the simulation could be much larger than the period of the vibration. We refer this approach the SBLA. However, there are some limitations of using this approximation. When the frequency is not very high, the boundary layer thickness $\delta$, i.e., $\delta=\sqrt{v_{m} / \pi f}$, is not small enough. The use of this boundary condition could be erroneous (Yu et al., 2006). In this report, DNS was used for low-frequency vibrations ( 3 and $5 \mathrm{~Hz}$ ), while SBLA was used for the higher frequencies $(10$ and $20 \mathrm{~Hz})$.

The above governing equations and boundary conditions are discretized by a finite volume method, and the resultant differential/algebraic equations are solved by DASPK solver with adaptive step size control (Lan and Liang, 1998). The total number of unknowns after the finite volume approximation is 34052 , and all the calculations are performed in a personal computer (P4-3 GHz CPU).

\section{Results and discussion}

\subsection{Interface morphology}

Figs. 2a and $\mathrm{b}$ show the interface shape evolution during Bridgman growth of SCN containing $0.064 \mathrm{wt} \%$ acetone and 
$0.15 \mathrm{wt} \%$ salol, respectively, at the pulling speed of $1.6 \mu \mathrm{m} / \mathrm{s}$. For acetone, at stationary, the interface was flat due to the nearly equal thermal conductivities of the melt and the crystal. As the solidification started, the interface moved downward and slightly deformed. At around $40 \mathrm{~min}$, a depression occurred at the center due to the local accumulation of acetone. Such a depression having a cusp tip broke down, due to constitutional supercooling, at about the same time. Further breakdown and enlargement of the pattern can be seen from the photographs at 60 and $80 \mathrm{~min}$, respectively.

The evolution of interface morphology for SCN/salol showed a similar behavior. However, as shown in Fig. 2b, the depression shape was wider. The morphological breakdown occurred at about the same time being about $40 \mathrm{~min}$. The bottom of the breakdown area was much flatter than that of SCN/acetone. We have enlarged the interface shapes at $60 \mathrm{~min}$ of both cases for comparison in Fig. 3. As shown, the shapes of the breakdown area were quite different. As will be explained later by numerical simulation, this was cased by the different solutal distributions at the interface.

Computer simulations were further carried as shown in Figs. $4 \mathrm{a}$ and $\mathrm{b}$ for SCN/acetone and SCN/salol, respectively. In each plot, the left-hand side is the streamlines, and the righthand side the solutal fields (iso-concentrations). As shown in Fig. 4a, at stationary, the interface was flat and the convection near the interface was extremely weak. The upper cell was caused by the radial heating. As the solidification started, the interface became concave and the flow cell near the interface

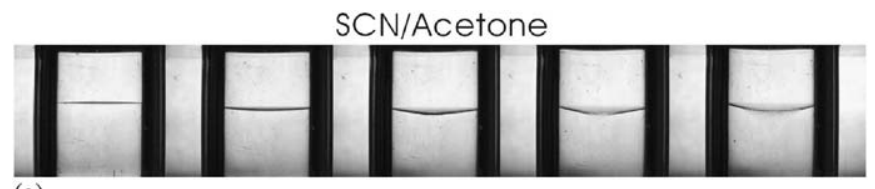

(a)

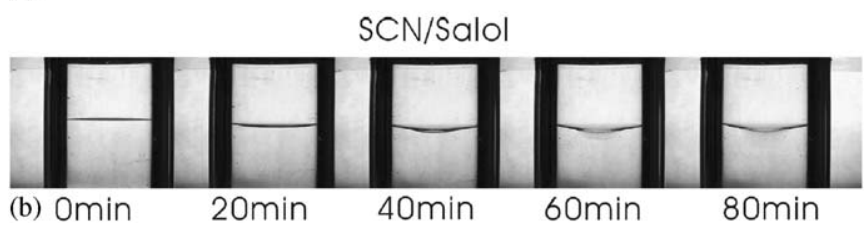

Fig. 2. Interface evolution of vertical Bridgman growth of SCN containing: (a) $0.064 \mathrm{wt} \%$ of acetone; (b) $0.15 \mathrm{wt} \%$ of salol. The ampoule pulling speed is $1.6 \mu \mathrm{m} / \mathrm{s}$.

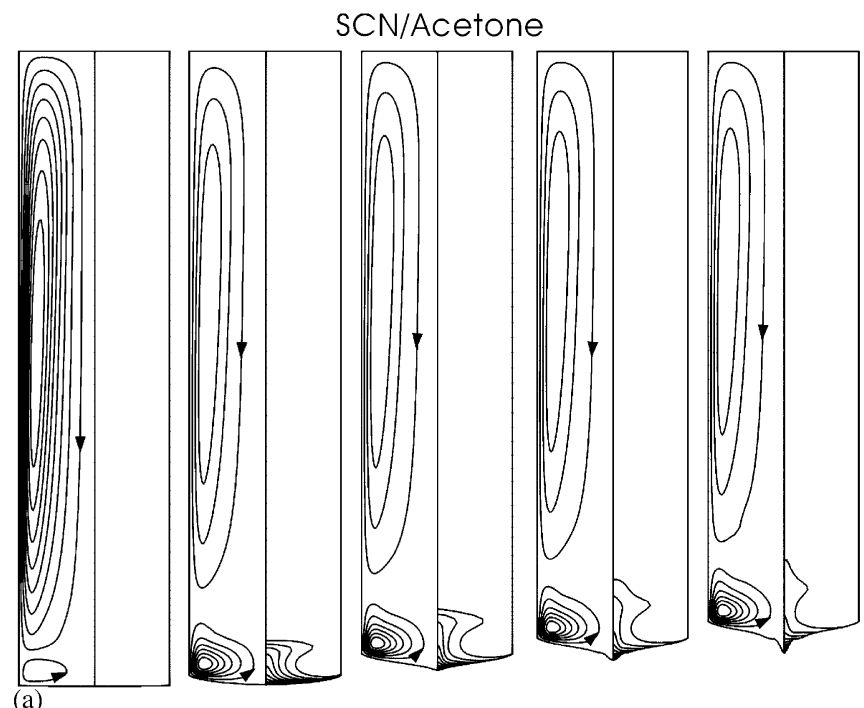

(a)
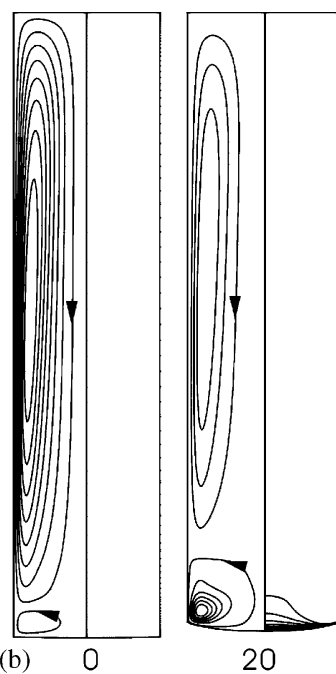

SCN/Salo
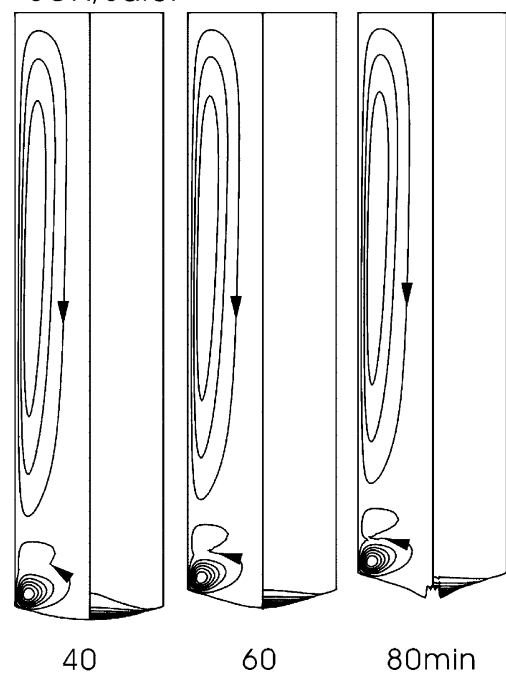

Fig. 4. Calculated flow and solute fields: (a) SCN/acetone; (b) SCN/salol; the ampoule pulling speed is $1.6 \mu \mathrm{m} / \mathrm{s}$. In (a) at $t=0, \Psi_{\min }=-1.8 \times 10^{-4} \mathrm{~g} / \mathrm{s}$, $\Psi_{\max }=3.037 \times 10^{-5} \mathrm{~g} / \mathrm{s}$, and $C / C_{0}=1$ and at $t=1 \mathrm{~h}, \Psi_{\min }=-1.8 \times 10^{-4} \mathrm{~g} / \mathrm{s}$, $\Psi_{\max }=3.037 \times 10^{-5} \mathrm{~g} / \mathrm{s}$, maximum $C / C_{0}=16.2693$, and minimum $C / C_{0}=1$. In (b) $t=0, \Psi_{\min }=-1.798 \times 10^{-4} \mathrm{~g} / \mathrm{s}, \Psi_{\max }=3.110 \times 10^{-5} \mathrm{~g} / \mathrm{s}$, and $C / C_{0}=1$; at $t=2400 \mathrm{~s}, \Psi_{\min }=-1.489 \times 10^{-4} \mathrm{~g} / \mathrm{s}, \Psi_{\max }=2.500 \times 10^{-4} \mathrm{~g} / \mathrm{s}$, maximum $C / C_{0}=6.0851$, and minimum $C / C_{0}=1$; at $t=4800 \mathrm{~s}$, $\Psi_{\min }=-1.486 \times 10^{-4} \mathrm{~g} / \mathrm{s}, \quad \Psi_{\max }=2.534 \times 10^{-4} \mathrm{~g} / \mathrm{s}, \quad$ maximum $C / C_{0}=16.145$, and minimum $C / C_{0}=1$.
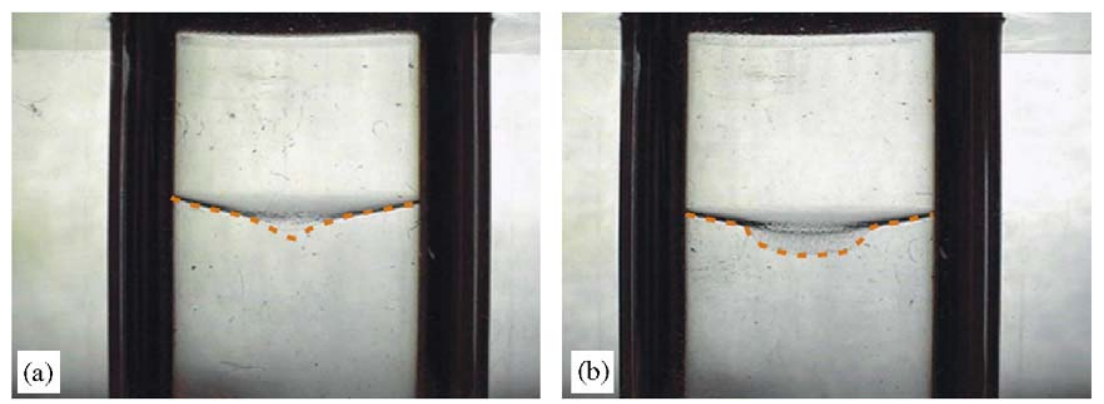

Fig. 3. Enlarged photograph and the sketch of the interface and the lower boundary of the breakdown morphology: (a) SCN/acetone; (b) SCN/salol. 


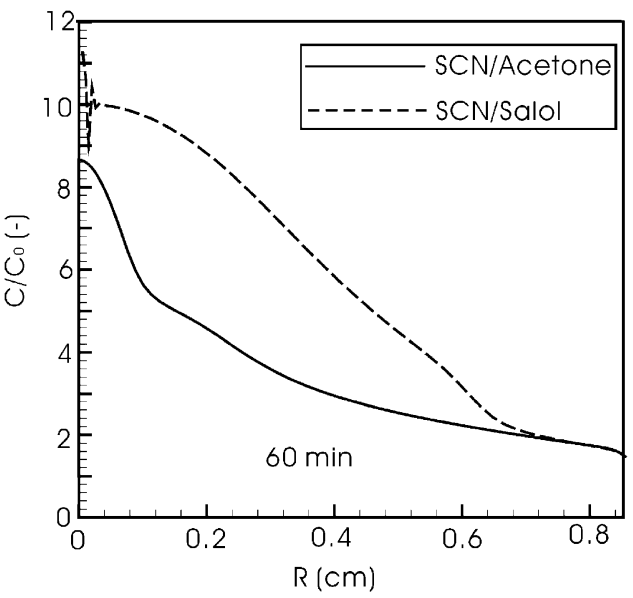

Fig. 5. Comparison of calculated radial solute segregations of different solutes at the growth interface; $t=60 \mathrm{~min}$. The wiggle of the radial salol concentration near $r=0$ was due to numerical breakdown caused by the large supercooling.

was enhanced. As a result, the acetone rejected during solidification was redistributed by the flow having an increasing acetone concentration toward the center of the interface. Because acetone is lighter than SCN, the radial acetone gradients also enhance the flow leading to a highly localized solute distribution at the center of the interface. The local acetone accumulation further caused a suppression of the interface there, which became obvious at about $40 \mathrm{~min}$. As will be discussed shortly through calculations, constitutional supercooling also occurred at about $35 \mathrm{~min}$. As the supercooling was established, morphological breakdown could occur when the supercooling overcame the interfacial energy. The simulated results at 60 and 80 min show a deep depression at the interface center, where high constitutional supercooling exists. However, in reality, the microscopic planar interface could no longer exist, as those shown in Fig. 2a. The supercooled interface has a cellular or dendritic structure. Because of the morphological breakdown, the accumulated acetone in front of the interface was trapped and the supercooling was reduced. However, the present simulation is not able to take this into account. Therefore, the simulation results after the morphological breakdown are only qualitative.

For SCN/salol, the convection near the interface was much weaker, as shown in Fig. 4b. Such a weaker flow was due the heavier solute, which suppressed the flow. In other words, the radial density due to thermal gradients was counter balanced by the solutal gradients; one could observe this contribution from the source term of Eq. (1). In addition, the flow cell near the interface was closer to the ampoule wall as compared with that in Fig. 4a. More importantly, the concentration profile was quite uniform near the interface, i.e., the much flatter isoconcentration lines. This also indicated the convective effect on the solute transport was much weaker.

The radial solute concentrations at 60 min from Fig. 4 were plotted in Fig. 5 for further comparison. As shown, the distribution near the interface center for $\mathrm{SCN} / \mathrm{salol}$ was much wider than that for SCN/acetone. The solute distributions happened to

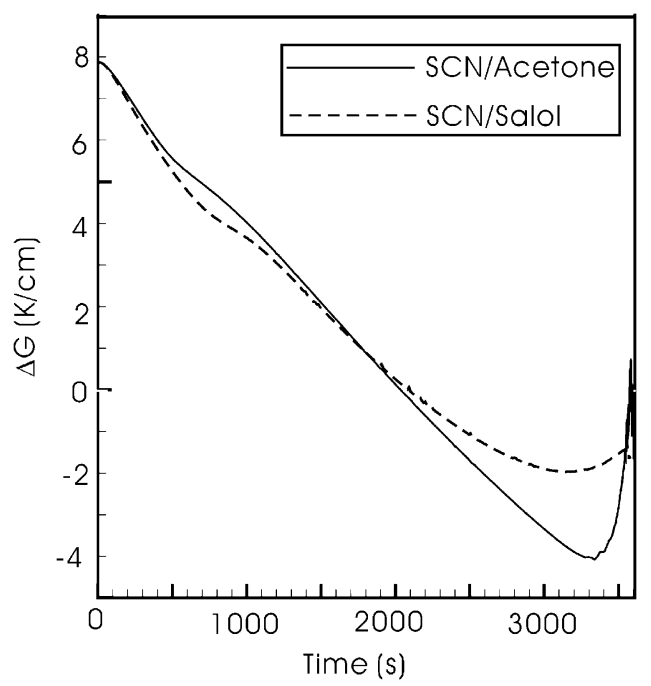

Fig. 6. Calculated constitutional superheating at the center of the interface for SCN/acetone and SCN/salol; constitutional supercooling occurs when $\Delta G<0$.

be consistent with the shapes of the morphological breakdown shown in Fig. 3. Moreover, because the convection near the solidification interface in $\mathrm{SCN} /$ salol was weaker, this caused the poorer global salol mixing there. As a result, the accumulation of salol was more than that of acetone.

To illustrate the onset of the constitutional supercooling, we extracted the superheating gradient from the previous simulated results as shown in Fig. 6. In the figure, the superheating gradient $\Delta G$ is defined as follows:

$\Delta G=\mathrm{d} T / \mathrm{d} n-m \mathrm{~d} C / \mathrm{d} n$,

where $m$ is the slope of the liquidus line obtained from the phase diagram and $n$ the normal distance from the interface; the superheatings in Fig. 6 were at the center of the interface. At stationary, the solute is uniform, so that $\Delta G=\mathrm{d} T / \mathrm{d} z$ is the thermal gradient at the interface (in the melt side). As shown in Fig. 6, the superheating gradients for both cases decreased monotonically with time. However, constitutional supercooling occurred slightly earlier for SCN/acetone. The earlier onset of the supercooling for SCN/acetone was attributed to the local acetone accumulation at the depression area as a result of the convection. The simulated results having a large supercooling were often accompanied by numerical breakdown at the interface. Therefore, the simulated results after $60 \mathrm{~min}$ were not reliable.

\subsection{Effect of angular vibration}

To further control the solute field and interface morphology, angular vibration was applied. The effect of vibration frequency on the interface morphology at $60 \mathrm{~min}$ after the ampoule translation is shown in Figs. 7a and b, for SCN/acetone and SCN/salol, respectively. For SCN/acetone in Fig. 7a, there was no morphological breakdown in $60 \mathrm{~min}$ for the frequency greater than $3 \mathrm{~Hz}$; at $3 \mathrm{~Hz}$, the interface was not quite smooth. Also, from 0 to $5 \mathrm{~Hz}$, the interface concavity decreased with 


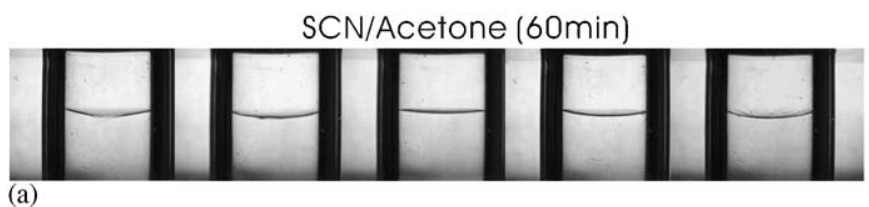

(a)

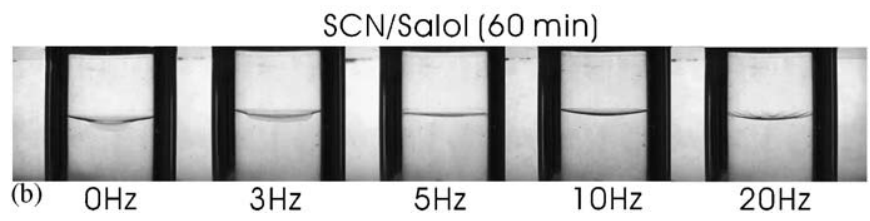

Fig. 7. Effects of angular vibration frequency on the interface shape and morphological instability at $60 \mathrm{~min}$ : (a) SCN/acetone; (b) SCN/salol.

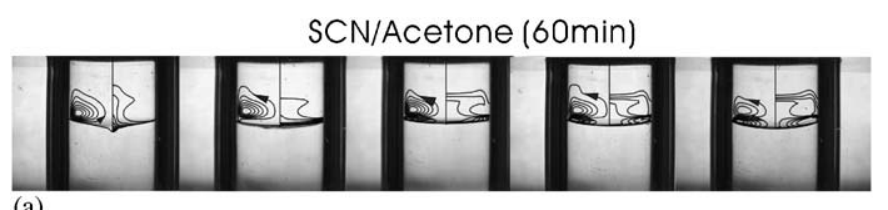

(a)

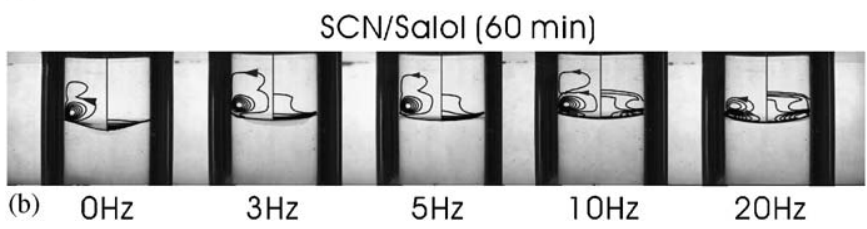

Fig. 8. Simulated flow and solute fields and the comparison with the observed interface at different vibration frequency at $60 \mathrm{~min}$ : (a) SCN/acetone; (b) $\mathrm{SCN} /$ salol.

the vibration frequency. However, the interface concavity increased again from 5 to $20 \mathrm{~Hz}$. Interestingly, a wavy interface was found at $20 \mathrm{~Hz}$, and the variation was along the angular direction. The effect of vibration for $\mathrm{SCN} /$ salol had a similar trend as shown in Fig. 7b. However, at $3 \mathrm{~Hz}$, the area of the morphological breakdown was significantly larger as compared with that of no vibration. The interface concavity also decreased from 3 to $5 \mathrm{~Hz}$, and then increased again from 5 to $20 \mathrm{~Hz}$. At $20 \mathrm{~Hz}$, a wavy interface was observed as well.

Computer simulation was further carried out, and the comparison with the interface was shown in Figs. 8a and b for SCN/acetone and SCN/salol, respectively. As shown, the simulated interface concavity for both cases agreed quite well with the experiments. It should be noticed that the interface was at the upper boundary of the breakdown area. More importantly, from 0 to $5 \mathrm{~Hz}$, the interface concavity decreased with the frequency, while from 5 to $20 \mathrm{~Hz}$, the concavity increased with the frequency. The reason is quite clear from the simulation. From 0 to $5 \mathrm{~Hz}$, the flow above the interface was weakened by vibration because of the radial outward streaming flow induced by the angular vibration. As a result, the solute distribution became more uniform and this reduced the interface concavity cased by the local solute accumulation. On the other hand, from 5 to $20 \mathrm{~Hz}$, the Schlichting flow became dominant. Since the flow was in clockwise direction and the isotherms were distorted with the flow, the interface concavity increased with the vibration intensity (frequency).
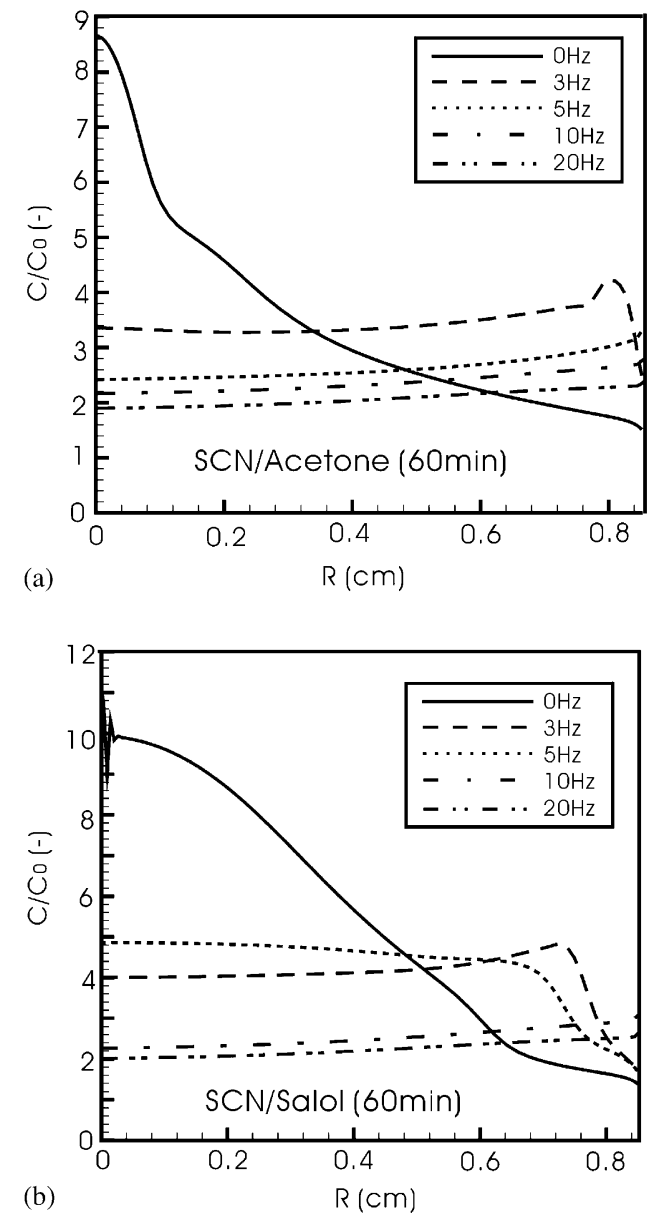

Fig. 9. Effect of angular vibration on the radial solute segregation: (a) SCN/acetone; (b) SCN/salol.

The radial solute concentrations extracted from the simulated results in Fig. 8 were plotted in Figs. 9a and b for SCN/acetone and SCN/salol, respectively. As shown, for both cases, the radial segregation reversed from 0 to $5 \mathrm{~Hz}$. This indicated that the Schlichting streaming flow was strong enough to overcome the buoyancy force and was able to push the solute from the interface center to the rim. From both Figs. 8 and 9, it was clear that when the frequency was greater than $10 \mathrm{~Hz}$, the Schlichting flow dominated and the solutal effect became insignificant, which could be seen from the solutal fields as well as the radial solute segregation profiles.

Fig. 10 shows the effect of the vibration frequency on the superheating gradient. As shown, in $1 \mathrm{~h}$, it appeared a large supercooling for $0 \mathrm{~Hz}$ and a small supercooling for $3 \mathrm{~Hz}$ at the interface center. Both were consistent with the observed result; the interface for $3 \mathrm{~Hz}$ had a little breakdown area in $1 \mathrm{~h}$, as shown in Fig. 7a. For SCN/salol, the supercooling occurred for the frequency being less than $10 \mathrm{~Hz}$. However, in the experiment, as shown in Fig. 7b, we did not observed a clear interface breakdown at the interface center for $5 \mathrm{~Hz}$. Instead, the morphological breakdown for $5 \mathrm{~Hz}$ occurred near the rim of the interface. As shown in Fig. 11, there was no supercooling for the frequency greater than $10 \mathrm{~Hz}$. 


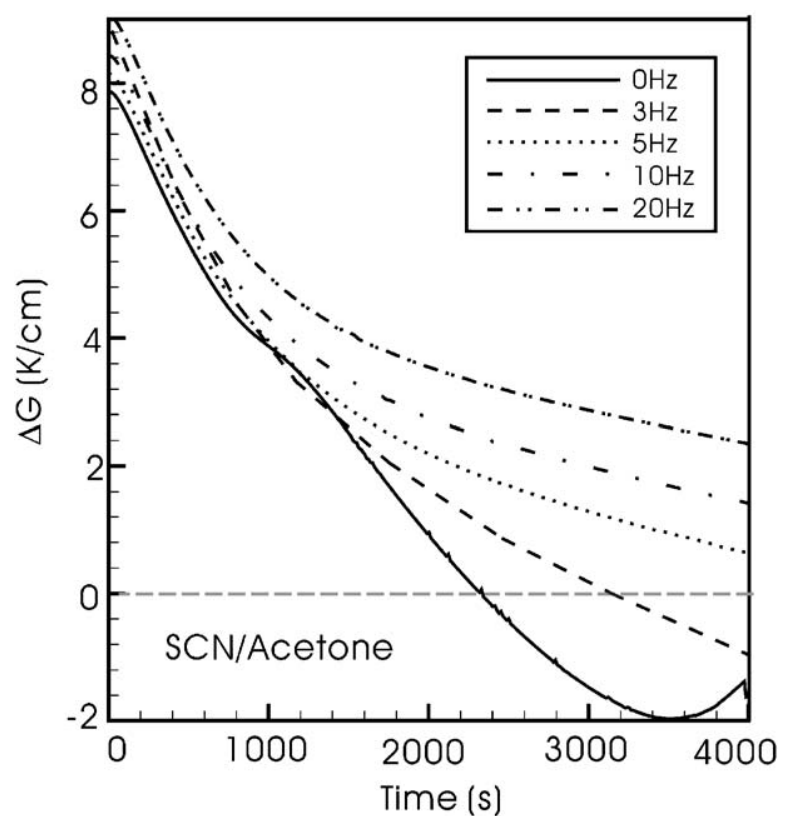

Fig. 10. Effect of angular vibration on the calculated constitutional superheating at the center of the interface for SCN/acetone; constitutional supercooling occurs when $\Delta G<0$.

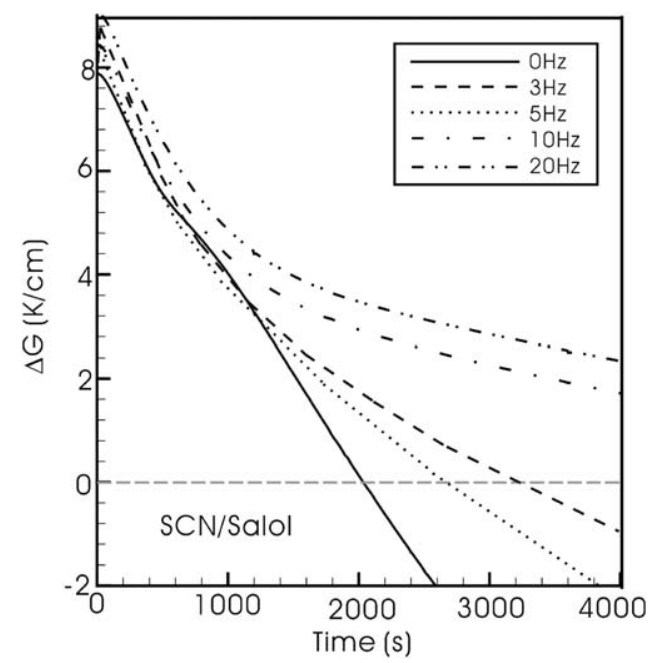

Fig. 11. Effect of angular vibration on the calculated constitutional superheating at the center of the interface for SCN/salol; constitutional supercooling occurs when $\Delta G<0$.

We also monitored the onset of supercooling at different places of the interface for $3 \mathrm{~Hz}$. Fig. 12 shows the evolution of the superheating gradients at three places of the interface for SCN/acetone. An observed interface at $80 \mathrm{~min}$ is shown as well. Interestingly, the area of the breakdown (from a to b) was consistent with the simulation (having a negative $\Delta G$ at about $80 \mathrm{~min}$ ). Similarly, the evolution of the superheating at three different places of the interface for SCN/salol is shown in Fig. 13. Again, the simulation also predicted the supercooled area (from a to b) correctly.

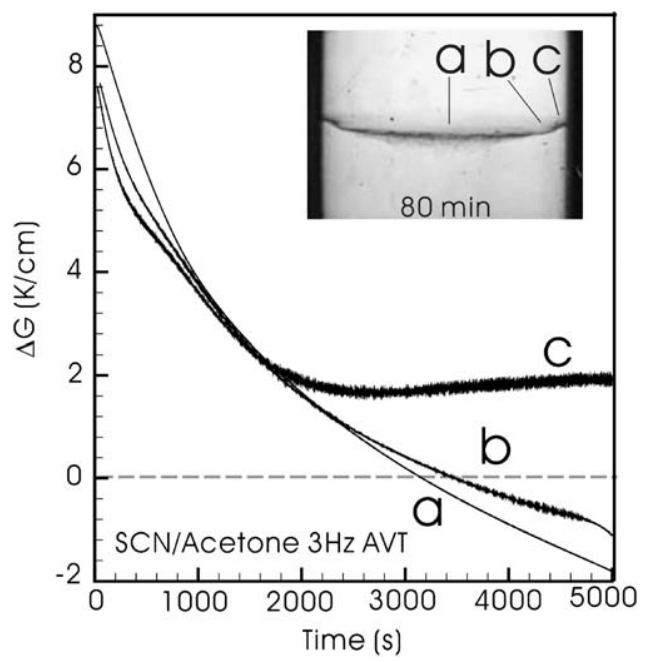

Fig. 12. Evolution of the superheating gradients at three different places of the interface for $\mathrm{SCN} /$ acetone at $3 \mathrm{~Hz}$.

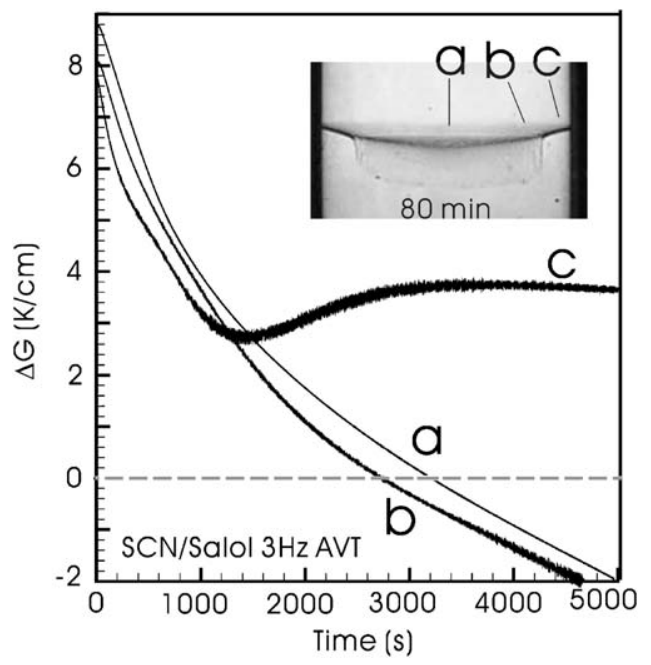

Fig. 13. Evolution of the superheating gradients at three different places of the interface for SCN/salol at $3 \mathrm{~Hz}$.

\section{Conclusion}

The effect of thermal-solutal flows on the interface shape and morphology in a vertical Bridgman crystal growth was investigated using succinonitrile alloys. Both lighter (acetone) and heavier (salol) solutes were considered. Based on the interface shape and its breakdown pattern, it was clear that the heavier solute (salol) significantly suppressed the flow near the growth interface. Computer simulation was further carried out and the simulated results were in good agreement with experimental observations. To further control the solute segregation, angular vibration was applied and the effect of frequency was investigated. Clearly, the induced Schlichting flow significantly modified the flow near the interface. As a result, the constitutional supercooling was effectively removed by vibration. At low frequencies, the interface concavity was reduced with the increasing frequency due to the solute redistribution. However, 
at high frequency, the interface concavity increased with the frequency due to the enhanced convective heat transfer by the Schlichting flow. The simulation also predicted reasonably well the area of the morphological breakdown based on the constitutional supercooling.

\section{Notation}

\begin{tabular}{|c|c|}
\hline$b_{v}$ & vibration amplitude \\
\hline$C$ & solute concentration \\
\hline$C_{p}$ & specific heat \\
\hline$D$ & solute diffusivity in the melt \\
\hline $\boldsymbol{e}_{z}$ & unit vector in $z$-direction \\
\hline$f$ & vibration frequency \\
\hline$g_{0}$ & gravitational acceleration \\
\hline$h_{c}$ & height of growth front \\
\hline$\Delta H$ & heat of fusion \\
\hline$k$ & thermal conductivity \\
\hline$L$ & length of ampoule \\
\hline$m$ & slope of the liquidus line in the phase diagram \\
\hline$n$ & normal distance from the interface \\
\hline $\operatorname{Pr}$ & Prandtl number, $v_{m} / \alpha_{m}$ \\
\hline$r$ & cylindrical coordinate \\
\hline$R_{c}$ & radius of crystal \\
\hline$R a_{S}$ & solutal Rayleigh number, $\beta_{s} R_{c}^{3} g_{0} C_{0} / v_{m} \alpha_{m}$ \\
\hline$R a_{T}$ & thermal Rayleigh number, $\beta_{T} R_{c}^{3} g_{0} T_{m} / v_{m} \alpha_{m}$ \\
\hline$S c$ & Schmidt number, $v_{m} / D$ \\
\hline$t$ & time \\
\hline$T$ & temperature \\
\hline$T_{\text {eff }}$ & effective heater temperature \\
\hline$T_{m}$ & melting point \\
\hline$u$ & $r$-component of velocity \\
\hline$u_{t}$ & $\begin{array}{l}\text { tangential velocity at the Schlichting boundary } \\
\text { layer }\end{array}$ \\
\hline$v$ & $z$-component of velocity \\
\hline$v_{\theta}$ & azimuthal velocity \\
\hline$z$ & cylindrical coordinate \\
\hline
\end{tabular}

\section{Greek letters}

$\begin{array}{ll}\alpha & \text { thermal diffusivity } \\ \beta_{S} & \text { solutal expansion coefficient } \\ \beta_{T} & \text { thermal expansion coefficient } \\ \delta & \text { Schlichting boundary layer thickness } \\ \mu_{m} & \text { viscosity } \\ v_{m} & \text { kinematic viscosity, } \mu_{m} / \rho_{m} \\ \rho & \text { density } \\ \varphi & \text { the angle between the tangent to the growth front } \\ \psi & \text { and the axis of rotation } \\ \omega & \text { stream function } \\ \Omega & \text { vorticity }\end{array}$

\section{Superscript}

* dimensionless variables

\section{Subscripts}

$0 \quad$ initial value

amp ampoule

$\begin{array}{ll}c & \text { crystal } \\ m & \text { melt } \\ \max & \text { maximum } \\ \min & \text { minimum }\end{array}$

\section{Acknowledgment}

This work was supported by the National Science Council (NSC) of the Republic of China, the Russian Foundation for Basic Research, and the Orchid Program between NSC and the PIA of France.

\section{References}

Adornato, P.M., Brown, R.A., 1987. Convection and segregation in directional solidification of dilute and non-dilute alloys: effect of ampoule and furnace design. Journal of Crystal Growth 80, 155-190.

Brice, J.C., 1986. Crystal Growth Processes. Wiley, New York.

Ghorayeb, K., Khallouf, H., Mojtabi, A., 1999. Onset of oscillatory flows in double-diffusive convection. International Journal of Heat Mass Transfer 42 (4), 629-643.

Lan, C.W., 2004. Recent progress of crystal growth modeling and growth control. Chemical Engineering Science 59, 1437-1457.

Lan, C.W., 2005. Flow and segregation control by accelerated rotation for vertical Bridgman growth of cadmium zinc telluride: ACRT versus vibration. Journal of Crystal Growth 274, 379-386.

Lan, C.W., Chen, F.C., 1996. A finite-volume method for solute segregation in directional solidification and comparison with a finite-element method. Computer Methods in Applied Mechanics and Engineering 31, 191-207.

Lan, C.W., Liang, M.C., 1998. Modeling of dopant segregation in vertical zone-melting crystal growth. Journal of Crystal Growth 186, 203-213.

Lan, C.W., Tu, C.Y., 2001. Morphological instability due to double diffusive convection in directional solidification: the pit formation. Journal of Crystal Growth 220, 619-630.

Monberg, E., 1994. Bridgman and related growth techniques. In: Hurle, D.T.J. (Ed.), Handbook of Crystal Growth 2a: Basic Techniques. North-Holland, Amsterdam, pp. 51-97.

Mullins, W.W., Sekerka, R.F., 1964. Stability of a planar interface during solidification of a dilute binary alloy. Journal of Applied Physics 35, 444-451.

Nishimura, T., Wakamatsu, M., Morega, A.M., 1998. Oscillatory doublediffusive convection in a rectangular enclosure with combined horizontal temperature and concentration gradients. International Journal of Heat and Mass Transfer 41 (11), 1601-1611.

Scheel, H.J., 1971. Flux growth of large crystals by accelerated cruciblerotation technique. Journal of Crystal Growth 13, 304-306.

Shi, K., Lu, W., 2006. Time evolution of double-diffusive convection in a vertical cylinder with radial temperature and axial solutal gradients. International Journal of Heat and Mass Transfer 49, 995-1003.

Singh, N.B., Mani, S.S., Adam, J.D., Coriell, S.R., Glicksman, M.E., Duval, W.M.B., Santaro, G.J., DeWitt, R., 1996. Direct observations of interface instability. Journal of Crystal Growth 166, 364-369.

Tiller, W.A., Jackson, K.A., Rutter, J.W., Chalmers, B., 1953. The redistribution of solute atoms during the solidification of metals. Acta Metallurgica 1, 428-437.

Yu, W.C., Chen, Z.B., Hsu, W.T., Roux, B., Lyubimova, T.P., Lan, C.W., 2004. Reversing radial segregation and suppressing morphological instability during Bridgman crystal growth by angular vibration. Journal of Crystal Growth 271, 474-480.

Yu, W.C., Chen, Z.B., Hsu, W.T., Roux, B., Lyubimova, T.P., Lan, C.W., 2006. Effects of angular vibration on the flow, segregation, and morphology in vertical Bridgman crystal growth. International Journal of Heat Mass Transfer, in press.

Zhou, H., Zebib, A., 1994. Oscillatory double-diffusive convection in crystal growth. Journal of Crystal Growth 135 (3-4), 587-593. 